\title{
Éditorial : Recherche sur la conduite automobile des personnes âgées : des preuves afin de guider le changement*
}

\section{Contexte}

Aux $20^{\mathrm{e}}$ et $21^{\mathrm{e}}$ siècles, la capacité à conduire une automobile est devenue étroitement liée à la mobilité dans les communautés. Les infrastructures publiques, les types de logement, tout autant que les trottoirs sont aménagés par rapport aux routes automobiles. On attend des personnes de tous âges de pouvoir conduire ; la perte de cette capacité apporte avec elle, non seulement une perte d'autonomie, mais aussi un déclin de santé et d'accès à la communauté. Dans son étude sur les habitudes de transport des personnes âgées au Canada, Turcotte (2012) a constaté que les personnes âgées sont extrêmement dépendantes de leurs véhicules, que le nombre de conducteurs âgés de plus de 65 ans va continuer à augmenter, et que «la majorité des personnes âgées n'ont pas l'intention de se déplacer, mais l'intention de rester là où elles vivent aussi longtemps que possible» (p. 15). Avec le vieillissement de la population, la proportion de personnes âgées qui conduira augmentera. Cela peut avoir des répercussions à plusieurs niveaux, y compris sur la politique, la santé et la sécurité publique, puisque les approches passées et actuelles de la conduite se sont concentrées sur les jeunes conducteurs.

Historiquement, la santé et le vieillissement n'ont pas été des sujets d'intérêt pour la politique et le développement de l'infrastructure automobile. L'accent a été traditionnellement mis sur la compétence et la connaissance en matière de conduite, en supposant ainsi que la santé, le fonctionnement physique et cognitif étaient intacts. Cependant, il est bien connu que les maladies et les conditions médicales chroniques peuvent affecter l'aptitude à conduire (Charlton et al, 2010; Marshall, 2008), et cet effet sera probablement plus grand au fur et à mesure que le nombre de conducteurs âgés augmentera.

La conduite est une activité fonctionnelle qui correspond bien à la Classification internationale du fonctionnement, du handicap et de la santé (CIF), élaborée par l'Organisation Mondiale de la Santé (2013). La conduite est influencée non seulement par des facteurs de santé (par exemple, des troubles physiques, cognitifs et de comportement), mais aussi par des facteurs personnels (par exemple, l'expérience en tant que conducteur / conductrice et la nécessité de conduire), les facteurs environnementaux (par exemple, le milieu rural par opposition à la vie urbaine, la conception des routes et la conception du véhicule), et les facteurs climatiques (par exemple, les conditions de conduite en hiver, en particulier au Canada). La perte de ce moyen affecte notre capacité à participer et à accéder à la communauté, ce qui peut entraîner l'isolement et les conséquences inhérentes de l'isolement.

Bien que l'accent ait été mis sur l'aptitude d'une personne âgée à conduire en fonction de son diagnostic ou

\footnotetext{
* Ces études ont été financées par une subvention de l'Équipe des Instituts de recherche en santé du Canada (IRSC) intitulée «L'Équipe des IRSC sur la conduite chez les aînés (Candrive II) Programme de recherche» (subvention 90429). Un soutien supplémentaire a été fourni par l'Ottawa Hospital Research Institute et Toronto Rehabilitation Institute, University Health Network. Gary Naglie est soutenu par George, Margaret et Gary Hunt Chaire de Famille en Médicine Gérontologique. Michel Bédard a été soutenu, au cours de la phase de développement de cette étude, par une Chaire de Recherche du Canada sur le Vieillissement et la Santé. Oxcandrive a été financé par une subvention de liaison en partenariat avec La Trobe University, VicRoads, le Département de justice de l'État de Victoria, la police de l'État de Victoria, le Transport Accident Commission, Road Safety Trust New Zealand, et Eastern Health. Les enquêteurs de Candrive remercient Lynn MacLeay, Candrive Program Manager, pour son rôle dans la gestion et l'opéralisation de l'étude sur les sites canadiens. Les enquêteurs de Candrive remercient également les associés de recherche pour leur dévouement et leur contribution à la réussite de l'étude (dans l'ordre $\mathrm{du}$ recrutement des participants): Candrive Research Coordinators Jennifer Biggs et Anita Jessup (Ottawa Coordinating Centre); Phyllis McGee (Victoria); Linda Johnson et Joanne Parsons (Winnipeg); Novlette Fraser et Sue Woodward (Toronto); Shneila Garrett (Hamilton); Felice Mendelsohn, Minh-Thy Dinh Truong, Suzie Schwartz et Rivi Levkovich (Montréal); et Laura Morrison et Hillary Maxwell (Thunder Bay). Un grand merci au personnel de soutien supplémentaire pour leur dévouement et leur contribution à la réussite de l'étude: Kelly Weegar et Chantal Rockwell (Ottawa). Les chercheurs de Candrive remercient également les participants à l'étude de la cohorte Candrive pour leur dévouement.
}

Sans leur engagement, cette publication n'aurait pas été possible. 
de son état de santé, la recherche doit explorer les facteurs qui influencent la conduite et ce qui peut être changé afin de maintenir cette population de conducteurs / conductrices actifs / actives dans la communauté. L'équipe de la conduite chez les aînés (Candrive II)* des IRSC a été financée par les Instituts de recherche en santé du Canada pour étudier les conducteurs canadiens plus âgés ( $\mathrm{n}=928)$, dans une étude prospective multi-centrique sur sept sites (Marshall et al., 2013). En outre, l'étude Ozcandrive, située en Australie et en Nouvelle-Zélande, suit un protocole similaire avec 250 conducteurs âgés de plus. Candrive, qui approche sa conclusion, a été conçu pour répondre à de nombreuses questions concernant les conducteurs âgés, en fournissant des informations sur leurs habitudes de conduite et les changements dans la conduite au fil du temps, ainsi que la façon dont leur santé et leur choix de véhicule affectent leur conduite. Les articles contenus dans ce numéro spécial traitent de sujets importants pour les conducteurs canadiens âgés, l'objectif ultime étant de prolonger la période de conduite en sécurité pour cette population.

\section{Les objectifs du numéro spécial}

L'objectif de ce numéro spécial est de mettre en évidence la recherche sur les conducteurs âgés qui est pertinente pour les Canadiens. Cette recherche reflète des éléments complexes ainsi que les facteurs impliqués dans la prise de décisions quant à l'aptitude à conduire, sans oublier les politiques et la conception de l'environnement qui peuvent influencer la sécurité des conducteurs âgés. L'objectif est de démontrer l'interaction nécessaire entre la santé des conducteurs et ses changements au fil du temps, le comportement et les attitudes des conducteurs, les interventions médicales, et les environnements de conduite (y compris les conditions météorologiques et les véhicules), ainsi que les effets de la politique sur les conducteurs et la sécurité publique.

\section{L'organisation du numéro spécial}

Ce numéro spécial met l'accent sur la recherche concernant les conducteurs âgés. Sept des dix articles se basent directement sur les données de Candrive / Ozcandrive (Marshall, 2013). Les trois autres articles ont un rapport direct avec les conducteurs plus âgés et ont été écrits par des membres de l'équipe Candrive. Gagnon et al. établissent la pertinence et la similitude de la cohorte de l'étude Candrive à la population de conducteurs canadiens âgés par rapport à l’Enquête sur la santé dans les collectivités canadiennes-Vieillissement en santé (ESCC-HA) (Statistique Canada, 2010). Ceci est pertinent pour les résultats de l'étude Candrive, car elle donne à penser que les résultats peuvent refléter la population de conducteurs plus âgés au Canada.
Étant donné la nature prospective de l'étude Candrive, Jouk et al. ont pu explorer des sujets tels que l'effet des changements d'attitude et de confiance du ou de la conductrice et leurs conséquences sur les habitudes de conduite. À l'opposé, Rapoport et al. et Tuokko et al. examinent les effets de changements cognitifs et la santé globale, respectivement, sur la confiance à conduire et les croyances chez les conducteurs âgés. Dans leur recension systématique, Cameron et Rapoport montrent que l'utilisation de médicaments antidépresseurs chez les conducteurs âgés contribue potentiellement à un risque significativement accru d'implication dans les collisions.

L'un des avantages des études Ozcandrive / Candrive est que l'on pourrait non seulement recueillir des informations subjectives sur les attitudes des conducteurs et leurs comportements, mais on pourrait aussi confirmer formellement les habitudes de conduite grâce au suivi GPS électronique. Smith et al. explorent les effets des conditions météorologiques sur la conduite et démontrent que ces dernières combinées à la lumière du jour sont influentes, mais qu'il y a d'autres facteurs qui sont aussi probablement en jeu dans les habitudes de conduite. De même, Koppel et al. utilisent davantage l'observation directe du conducteur pour confirmer que la capacité de conduire est liée aux capacités de conduire perçues par les participants et la fréquence signalée de la conduite dans des situations difficiles. Vrkljan et al. et Kajaks et al. explorent l'interaction entre le conducteur et le véhicule. Vrkljan et al. confirment que la taille du conducteur et le sexe influencent le type et la taille du véhicule acheté. Dans une nouvelle simulation, Kajaks et al. sont en mesure de montrer qu'une gamme réduite de mouvement dans le cou, souvent présente chez les conducteurs âgés, peut influencer la capacité d'un conducteur à vérifier l'angle mort à gauche; cela a des implications directes sur la conception des véhicules. Cette recherche renforce la constatation qu'il y a plusieurs facteurs qui contribuent à la sécurité routière. C'est cette complexité qui conduit à la confusion et à l'incertitude quand des conducteurs, des familles et des praticiens de la santé doivent travailler ensemble pour examiner la possibilité de cesser de conduire en réponse aux questions de santé. Ces défis sont bien résumés par Mazer et al. qui examinent l'éthique de la déclaration des risques de conduite liés à la santé aux autorités délivrant des permis.

\section{Observations finales}

Les multiples facettes de la conduite, des conditions de la route pour les véhicules, et même les chauffeurs, ont continué à changer et à évoluer au fil des ans. Bien que la sécurité routière continue à être améliorée globalement, la recherche en cours sur les questions 
auxquelles les conducteurs âgés font face reste importante, car cette population de conducteurs / de conductrices croît le plus rapidement. Des facteurs tels que la santé et l'anticipation de devoir arrêter la conduite sont particulièrement pertinents pour les conducteurs âgés et nécessitent une étude plus approfondie dans le cadre du processus d'extension de la période de conduite sécuritaire pour les personnes âgées.

Shawn C. Marshall

The Ottawa Hospital Research Institute

Rédacteur invité

\section{Références}

Charlton, J., Koppel, S., Odell, M., Devlin, A., Langford, J., O'Hare, M., et al. (2010). Influence of chronic illness on crash involvement of motor vehicle drivers (2nd ed.). Report no. 300. Melbourne, Australia: Monash University Accident Research Centre.

Marshall, S. C. (2008). The role of reduced fitness to drive due to medical impairments in explaining crashes involving older drivers. Traffic Injury Prevention 9, 291-298.

Marshall, S. C., Man-Son-Hing, M., Bédard, M., Charlton, J., Gagnon, S., Gélinas, I., et al. (2013). Protocol for Candrive II/Ozcandrive, a multicentre prospective older driver cohort study. Accident Analysis \& Prevention (Candrive special issue), 61, 245-252.

Statistics Canada (2010). Canadian Community Health Survey CCHS HA-Healthy Aging (CCHS). User Guide. Ottawa, ON: Author. Retrieved from http://www23.statcan.gc.ca/ $\mathrm{imdb} / \mathrm{p} 2 \mathrm{SV}$.pl?Function=getSurvey\&SDDS=5146

Turcotte, M. (2012). Profile of seniors' transportation habits. In Canadian Social Trends (component of Statistics Canada Catalogue no.11-008). Ottawa, ON: Statistics Canada. http: / / www.statcan.gc.ca/pub/11-008x/2012001/ article/11619-eng.pdf

World Health Organization (2013). How to use the ICF: A practical manual for using the International Classification of Functioning, Disability and Health (ICF). (Exposure draft for comment). Geneva, Switzerland: Author. 